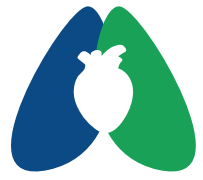

ASSOBRAFIR C I Ê N C I A

\section{Análise da força muscular respiratória de pacientes com Apneia Obstrutiva do Sono: uma revisão de literatura}

\author{
Analysis of respiratory muscle strength in patients with Obstructive \\ Sleep Apnea: a literature review
}

\author{
Breno Gomes Rodrigues da Silva ${ }^{1}$, Camila Danielle Cunha Neves ${ }^{1}$ (C) \\ ${ }^{1}$ Curso de Fisioterapia, Faculdade Sete Lagoas (FACSETE), Sete Lagoas, MG, Brasil
}

Como citar: Como citar: Silva BGR; Neves CDC. Análise da força muscular respiratória de pacientes com apneia obstrutiva do sono: uma revisão de literatura. ASSOBRAFIR Ciênc. 2021;12:e42420. https://doi. org/10.47066/2177-9333.AC.2020.0026

\begin{abstract}
Resumo
Introdução: a Apneia Obstrutiva do Sono (AOS) trata-se de um distúrbio respiratório, definido por episódios recorrentes de colapso da via aérea superior. Pesquisas mostram que a AOS podem levar ao comprometimento da força muscular respiratória, o qual, parece ter relação com a gravidade da doença. No entanto, ainda não há relatos na literatura que demonstrem as características da força muscular respiratória de pacientes com diferentes gravidades da AOS. Objetivo: revisar e descrever os impactos da AOS sobre a força muscular respiratória, bem como, analisar se existem diferenças conforme a gravidade da AOS. Métodos: Trata-se de uma revisão de literatura, na qual foi realizada a busca científica nas seguintes bases de dados eletrônicas: PubMed, SciELO, PEDro e Biblioteca Virtual em Saúde. A busca foi realizada através da combinação dos seguintes descritores em saúde: apneia obstrutiva do sono, força muscular e pressões respiratórias máximas e suas respectivas traduções para a língua inglesa. Resultados: foram selecionados seis artigos, dos quais três encontraram comprometimento da força muscular inspiratória em pacientes com AOS moderada à grave e três não encontraram esta relação. Somente um estudo incluiu indivíduos com o quadro leve da AOS. Conclusão: Os resultados publicados na literatura ainda são divergentes com relação ao impacto da AOS na força muscular respiratória, no entanto, estudos que demonstram fraqueza muscular sugerem que a AOS compromete a força muscular inspiratória de pacientes com AOS moderada à grave. Não existem dados suficientes para a força muscular respiratória no quadro leve da AOS.
\end{abstract}

Palavras-chave: Apneia Obstrutiva do Sono; Força Muscular; Pressões Respiratórias Máximas.

\begin{abstract}
Background: Obstructive Sleep Apnea (OSA) is a respiratory disorder, defined by recurrent episodes of upper airway collapse. Studies show that OSA can lead impaired respiratory muscle strength, which appears to be related to the severity of the disease. However, there are still no reports in the literature that demonstrate the characteristics of respiratory muscle strength in patients with different OSA severities. Aim: to review and describe the impacts of OSA on respiratory muscle strength, as well as to analyze whether there are differences according to the severity of OSA. Methods: It is a literature review, in which the scientific search was carried out in the following electronic databases: PubMed, SciELO, PEDro and Biblioteca Virtual em Saúde. The search was carried out by combining the following health descriptors: sleep apnea, obstructive; muscle strength and maximum respiratory pressures and their respective translations into Portuguese. Results: six studies were selected, of which three found impaired inspiratory muscle strength in patients with moderate to severe OSA, and three did not find this relation. Only one study included individuals with mild OSA. Conclusion: The results published in the literature are still divergent regarding the impact of OSA on respiratory muscle strength, however, studies that demonstrate muscle weakness suggest that OSA compromises the inspiratory muscle strength of patients with moderate to severe OSA. There are insufficient data for respiratory muscle strength in mild OSA.
\end{abstract}

Keywords: Sleep Apnea, Obstructive; Muscle Strength; Maximum Respiratory Pressures. 


\section{INTRODUÇÃO}

A Apneia Obstrutiva do Sono (AOS) trata-se de um distúrbio respiratório do sono, definido por episódios recorrentes de colapso da via aérea superior (VAS), ou seja, aumento da resistência à passagem do ar, seja ela parcial (hipopneia) ou total (apneia), induzido pelo sono ${ }^{1}$. Segundo a Academia Americana de Medicina do Sono, a AOS é caracterizada por episódios de obstrução do fluxo aéreo que duram pelo menos 10 segundos e que frequentemente são acompanhados de esforço respiratório, sem adequada ventilação, ocasionando a dessaturação da oxihemoglobina, hipercapnia e microdespertares, que colaboram com a privação do sono. Tais fatores contribuem para um sono não reparador, sonolência e fadiga excessivas durante o dia ${ }^{1}$.

Dados publicados recentemente demonstram que há uma estimativa de mais de 900 milhões de adultos no mundo com AOS, com idade entre 30 e 69 anos. Além disso, o Brasil foi apontado como o terceiro país com maior número de casos no mundo inteiro, com estimativa de prevalência de 33\% de pacientes com AOS no país².

A AOS é uma condição multifatorial, sendo mais prevalente em pessoas idosas, acima do peso, do sexo masculino e em indivíduos com alterações anatômicas craniofaciais, como a micrognatia que acarreta posteriorização da base da língua ${ }^{3}$. Estudos sugerem que os episódios de obstrução ocorrem em sua maioria durante o sono REM (do inglês rapid eye movement, "movimento rápido dos olhos"), momento em que ocorre a cessação do estímulo excitatório aos neurônios motores da VAS, frente a redução da noradrenalina e serotonina, acarretando em diminuição da atividade muscular ${ }^{4}$.

Estes fatores parecem influenciar na manutenção da patência da faringe durante o sono. Sendo assim, já foi demonstrado que durante o sono, pacientes com AOS apresentam um desbalanço entre as forças dilatadoras e constritoras da VAS, com maior redução do tônus dos músculos dilatadores da faringe quando comparados a sujeitos saudáveis. Dessa forma, pacientes com AOS apresentam aumento da pressão de fechamento da VAS durante o sono, o que acarreta colapso da faringe, na maior resistência a passagem do ar e em apneia ${ }^{5}$.

O diagnóstico da AOS é feito através do exame de polissonografia noturna, que durante o sono avalia parâmetros como frequência cardíaca, fluxo aéreo, oximetria, presença de movimentos torácicos, abdominais, ronco e o índice de apneia e hipopneia (IAH) por hora de sono, o qual permite avaliar a gravidade da AOS. Assim, a gravidade da AOS é classificada em leve quando o IAH for $\geq 5$ e $\leq 14,9$, moderada quando o IAH for $\geq 15$ e $\leq 29,9$ e grave quando o IAH for $>$ que 30 vezes por hora de sono ${ }^{6}$.

Conforme a classificação da gravidade da AOS, diversas opções de tratamento são disponibilizadas, tais como a terapia com aparelho oral, terapia posicional, perda de peso, modificações comportamentais e cirurgia reconstrutiva das VAS ${ }^{6}$. Entretanto, o mais utilizado é a terapia através de pressão positiva nas vias aéreas (CPAP)7.

Pesquisas mostram a eficácia da CPAP na atenuação da sonolência excessiva e melhora da qualidade de vida dos pacientes, embora, o efeito da CPAP na redução de eventos cardiovasculares adversos, tais como a ocorrência de infarto agudo do miocárdio ou acidente vascular encefálico, ainda não esteja bem definido na literatura ${ }^{8}$. No entanto, a adesão do paciente tem sido um fator limitante da terapêutica. Adicionalmente, muitos pacientes com AOS não têm acesso ao tratamento adequado, o que aumenta o risco de deficiências na estrutura e função do corpo?.

Neste contexto, alguns estudos têm demonstrado que pacientes com AOS grave podem apresentar o comprometimento da função da musculatura respiratória, caracterizada principalmente pela fraqueza muscular inspiratória ${ }^{9-11}$. Esta deficiência tem sido relacionada a lesão oxidativa dos músculos respiratórios, bem como, pela sobrecarga mecânica imposta sobre estes músculos ${ }^{12,13}$. A avaliação da força muscular respiratória é uma medida simples. Sua importância na prática clínica ocorre, uma vez que a fraqueza desses músculos, dependendo da gravidade, pode acarretar em hipoventilação alveolar, insuficiência respiratória, além de dispneia e intolerância aos esforços ${ }^{14,15}$

Dessa forma, estes achados demonstram que a AOS pode comprometer a função dos músculos respiratórios, a qual, parece ter relação com a gravidade da doença. No entanto, ainda não há relatos na literatura que demonstrem as características da força muscular respiratória de pacientes com diferentes gravidades da AOS. Portanto, o objetivo deste estudo é revisar e descrever os impactos da AOS sobre a força muscular respiratória, bem como, analisar se há diferença do comprometimento da função muscular respiratória conforme a gravidade da AOS.

\section{MÉTODOS}

Trata-se de uma revisão de literatura integrativa, na qual foi realizada a busca científica nas seguintes bases de dados eletrônicas: Medical Literature Analysis and Retrievel System Online (MEDILINE) via PubMed, Scientific Electronic Library Online (SciELO), Physiotherapy Evidence Database (PEDro) e Biblioteca Virtual em Saúde (BVS). A busca foi realizada através da combinação dos seguintes descritores em ciências da saúde (DeCS): apneia obstrutiva do sono, força muscular e pressões respiratórias máximas e suas respectivas traduções para a língua inglesa: sleep apnea, obstructive; muscle strength e maximal respiratory pressures.

Os critérios de inclusão adotados para este estudo foram: artigos sem limite de tempo, escritos na língua inglesa e portuguesa, estudos do tipo observacionais, descritivos, experimentais ou clínicos, que descrevessem dentre os seus objetivos o intuito de investigar ou avaliar a força muscular respiratória (inspiratória e/ou expiratória) de pacientes com AOS. Já os critérios de exclusão foram: revisões narrativas 
ou sistemáticas da literatura, cartas ao editor, diretrizes, trabalhos de conclusão de curso, dissertações ou teses; estudos que descrevessem a força muscular respiratória como um desfecho primário ou secundário para a análise dos efeitos de terapêuticas aplicadas em pacientes com AOS; e, estudos que não comparassem os resultados da força muscular respiratória de pacientes com AOS com um grupo controle ou com valores de referência.

A qualidade dos artigos foi descrita por meio do sistema Qualis/CAPES (sistema brasileiro de avaliação dos periódicos da Coordenação de Aperfeiçoamento de Pessoal de Nível Superior), em período de classificação do quadriênio 2013-2016. Para isso, analisou-se a classificação dos periódicos em que os artigos foram publicados, os quais são categorizados em estratos que variam de indicadores que vão de A1 (mais elevado) passando por A2, B1, B2, B3, B4, B5 e C (peso 0).

\section{RESULTADOS}

Inicialmente, foram encontrados 98 artigos. Foi realizada a leitura exploratória dos títulos e resumos, sendo excluídos 92 estudos por não se enquadrarem nos critérios de inclusão desta revisão. Sendo assim, foram selecionados seis estudos para a leitura do texto completo, conforme demonstrado na Figura 1.

Dos trabalhos elegidos, três avaliaram a força muscular inspiratória pelas medidas da pressão inspiratória máxima (PI máx.) e pressão transdiafragmática máxima (Pdi) e força muscular expiratória pela medida da pressão expiratória máxima (PE máx.) e pressão gástrica máxima (Pgas), e três avaliaram somente a força muscular inspiratória (PI máx). Os estudos selecionados foram publicados de 2006 a 2018, sendo encontrados quatro artigos na língua inglesa e dois na portuguesa. A Tabela 1 apresenta o resumo dos estudos analisados, contendo seus objetivos, características gerais das amostras, os principais resultados e o estrato Qualis/CAPES.

Observa-se que na sua maioria, os estudos apresentaram indivíduos do sexo masculino, adultos, com AOS moderada à grave, associada ou não a outras comorbidades, como obesidade e diabetes tipo II. Além disso, foram encontrados resultados conflitantes quanto a influência da AOS na força muscular respiratória. Com relação à qualidade dos artigos, nota-se que na sua maioria, estes foram publicados em periódicos que são classificados em estratos com indicadores mais elevados de qualidade (A1 e A2).

\section{DISCUSSÃO}

Esta revisão mostrou que a literatura científica dispõe de seis estudos que objetivaram avaliar a força muscular respiratória de pacientes com AOS, no entanto, seus resultados ainda são divergentes. Além disso, a maioria dos estudos encontrados demonstrou os resultados de pacientes com AOS moderada à grave, o que sugere que o comprometimento da força muscular respiratória pode estar associado à gravidade da doença.

A avaliação da força muscular respiratória é frequentemente realizada em pacientes com doenças neuromusculares e doenças respiratórias do trato respiratório inferior, no entanto, deve ser aplicada também em pacientes com doenças que afetam as vias aéreas superiores ${ }^{15}$, como a AOS. Essa prática é importante, visto que a fraqueza muscular respiratória pode contribuir para a redução dos volumes pulmonares, reduzindo assim a tolerância aos esforços e levando a piora da qualidade de vida desses indivíduos ${ }^{9}$.

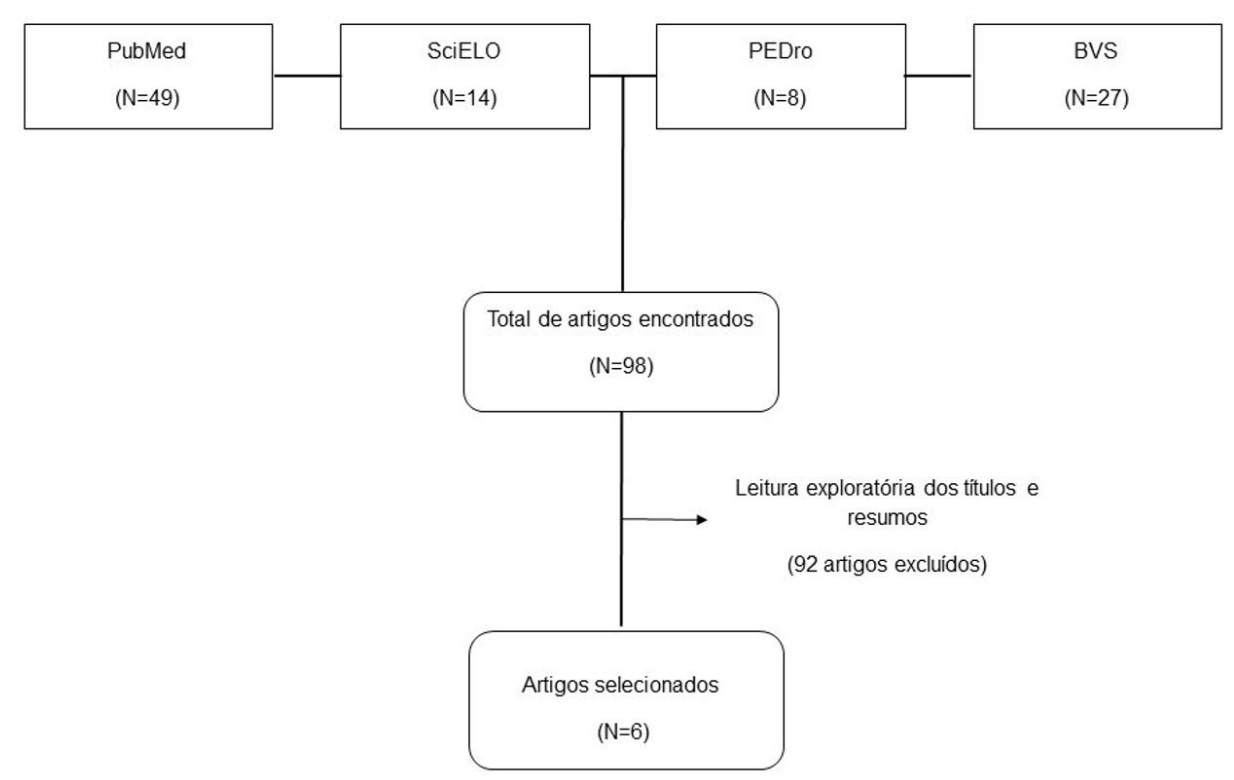

Figura 1. Fluxograma do processo de pesquisa e seleção dos artigos. 
‘Tabela 1. Resumo dos artigos que avaliaram a força muscular respiratória de pacientes com AOS leve, moderada e grave.

\begin{tabular}{|c|c|c|c|c|}
\hline Estudo & Objetivo & Amostra & Resultados & $\begin{array}{l}\text { Qualis/ } \\
\text { CAPES }\end{array}$ \\
\hline Carvalho et al., 2018 & $\begin{array}{l}\text { Avaliar a força muscular } \\
\text { respiratória (PImáx. e PEmáx.) } \\
\text { em indivíduos obesos com } \\
\text { AOS moderada e grave. }\end{array}$ & $\begin{array}{l}31 \text { indivíduos ( } 10 \text { homens e } 21 \\
\text { mulheres) com idade média de } \\
58 \text { anos, obesos (média de IMC } \\
\text { de } 31,2 \mathrm{~kg} / \mathrm{m} 2 \text { ). }\end{array}$ & $\begin{array}{l}\text { Identificou fraqueza muscular } \\
\text { inspiratória (PImáx) em } \\
\text { indivíduos com AOS de } \\
\text { moderada a grave, de acordo } \\
\text { com valores de referência. }\end{array}$ & $\mathrm{A} 2$ \\
\hline Rehling et al., 2017 & $\begin{array}{l}\text { Comparar a força muscular } \\
\text { inspiratória (Plmáx.) de } \\
\text { pacientes com diabetes tipo } 2 \\
\text { e AOS moderada e grave. }\end{array}$ & $\begin{array}{c}32 \text { indivíduos ( } 28 \text { homens e } 5 \\
\text { mulheres) no grupo com AOS } \\
\text { e diabetes, com idade média } \\
\text { de } 64 \text { anos. }\end{array}$ & $\begin{array}{l}\text { Identificou comprometimento } \\
\text { da musculatura inspiratória } \\
\text { (PImáx) em indivíduos com } \\
\text { AOS de moderada a grave, em } \\
\text { comparação com valores de } \\
\text { referência de uma população } \\
\text { pareada por sexo e idade. }\end{array}$ & $\mathrm{A} 2$ \\
\hline $\begin{array}{c}\text { Tassinari et al., } \\
2016\end{array}$ & $\begin{array}{l}\text { Comparar a força muscular } \\
\text { respiratória (PImáx. e PEmáx.) } \\
\text { entre sujeitos saudáveis e } \\
\text { pacientes com AOS.* }\end{array}$ & $\begin{array}{c}38 \text { indivíduos de ambos os } \\
\text { sexos, divididos em grupo } \\
\text { controle (=19) e grupo AOS } \\
\text { (=19), com idade média de } 49 \\
\text { anos. }\end{array}$ & $\begin{array}{l}\text { Não identificou } \\
\text { comprometimento da força } \\
\text { muscular respiratória (PImáx } \\
\text { e PEmáx), em indivíduos com } \\
\text { AOS, em comparação com o } \\
\text { grupo controle. }\end{array}$ & B3 \\
\hline Steier et al., 2010 & $\begin{array}{l}\text { Quantificar a atividade dos } \\
\text { músculos respiratórios em } \\
\text { pacientes com AOS. }\end{array}$ & $\begin{array}{l}42 \text { indivíduos, de ambos os } \\
\text { sexos, divididos em grupo AOS } \\
(\mathrm{N}=21) \text { e controle }(\mathrm{N}=21) \text {, com } \\
\text { idade média de } 44 \text { e } 36 \text { anos, } \\
\text { respectivamente. }\end{array}$ & $\begin{array}{l}\text { Não demonstrou diferença na } \\
\text { força muscular inspiratória, } \\
\text { obtida por meio da pressão } \\
\text { transdiafragmática máxima } \\
\text { (Pdi) e nem expiratória, por } \\
\text { meio da pressão gástrica } \\
\text { máxima (Pgas) em pacientes } \\
\text { com AOS em sua maioria } \\
\text { grave. }\end{array}$ & B1 \\
\hline Chien et al., 2010 & $\begin{array}{l}\text { Investigar o efeito da AOS na } \\
\text { força muscular inspiratória } \\
\text { (PImáx.) em pacientes com } \\
\text { AOS grave em comparação } \\
\text { com o grupo controle. }\end{array}$ & $\begin{array}{l}30 \text { indivíduos, do sexo } \\
\text { masculino, divididos em grupo } \\
\text { AOS }(N=15) \text { e controle }(N=15) \\
\text { com idade média de } 51 \text { anos. }\end{array}$ & $\begin{array}{c}\text { Demonstrou que pacientes } \\
\text { com AOS grave têm redução } \\
\text { da força muscular inspiratória } \\
\text { (PImáx.) }\end{array}$ & $\mathrm{A} 1$ \\
\hline $\begin{array}{l}\text { Shepherd et al., } \\
2006\end{array}$ & $\begin{array}{l}\text { Determinar a força muscular } \\
\text { inspiratória (PImáx.) } \\
\text { em indivíduos com AOS } \\
\text { clinicamente significativa (IAH } \\
\text { > 20/h). }\end{array}$ & $\begin{array}{l}94 \text { indivíduos ( } 52 \text { homens } \\
\text { e } 42 \text { mulheres) com média } \\
\text { de idade de } 47 \text { e } 49 \text { anos, } \\
\text { respectivamente }\end{array}$ & $\begin{array}{l}\text { Demonstrou que a força } \\
\text { muscular inspiratória (PImáx.) } \\
\text { não apresentou diferença } \\
\text { entre indivíduos com e sem } \\
\text { AOS moderada a grave. }\end{array}$ & $\mathrm{A} 1$ \\
\hline
\end{tabular}

PImáx.: Pressão inspiratória máxima; PEmáx.: pressão expiratória máxima; AOS: Apneia Obstrutiva do Sono; Pdi: Pressão transdiafragmática máxima; Pgas: pressão gástrica máxima; IAH: Índice de Apneia e Hipopneia. ${ }^{*}$ O estudo não mencionou a gravidade da AOS da amostra.

Os três estudos que encontraram fraqueza muscular respiratória ${ }^{9-11}$, concluíram que somente a PI máx. estava reduzida. O maior comprometimento foi observado no estudo feito por Chien et al. ${ }^{11}$, no qual o valor médio da PI máx. de indivíduos do grupo controle foi de 100,3 $\mathrm{CmH}_{2} \mathrm{O}$ e a média demonstrada pelos pacientes com AOS foi de $73,3 \mathrm{cmH}_{2} \mathrm{O}$, demonstrando assim, uma diferença de $27 \mathrm{cmH}_{2} \mathrm{O}$ entre os participantes. No estudo feito por Rehling et al. ${ }^{10}$, o valor predito médio da PI máx. era de $92,9 \mathrm{CmH}_{2} \mathrm{O}$ e a média demonstrada pelos pacientes foi de $77 \mathrm{cmH}_{2} \mathrm{O}$, o que representou uma redução de $17 \%$. Já, no estudo de Carvalho et al. ${ }^{9}$, os pacientes com AOS apresentaram valor médio de $\mathrm{PI}$ máx. de $60 \mathrm{cmH}_{2} \mathrm{O}$, valor este, que determina fraqueza muscular inspiratória na população estudada.

O maior comprometimento dos músculos inspiratórios pode estar relacionado à sobrecarga mecânica desta musculatura, visto que esses músculos são expostos a um esforço inspiratório contra uma via aérea obstruída ${ }^{13}$. A fisiopatologia da AOS está relacionada ao colapso da VAS diante da redução do tônus da faringe, levando assim, ao aumento da resistência à passagem de ar e da pressão intratorácica, que somadas, levam ao aumento da carga respiratória ${ }^{1,5}$. Dessa forma, os episódios de colapso da VAS são frequentemente acompanhados de esforço respiratório, considerando que para ocorrer a ventilação pulmonar adequada, os músculos inspiratórios precisam ultrapassar a carga respiratória, que nos pacientes com AOS, está aumentada ${ }^{1,5}$. Assim, os músculos inspiratórios tornam-se cada vez mais exigidos, o que a longo prazo, parece comprometer o seu desempenho.

Estes achados estão de acordo com o estudo feito por Barreiro et al. ${ }^{15}$ que encontraram níveis aumentados de carbonilação e nitração de proteínas nos músculos intercostais externos de pacientes com AOS, o que sugere aumento da lesão oxidativa dos músculos inspiratórios, 
acarretando em comprometimento da função desta musculatura. Segundo Rosenzweig et al. ${ }^{12}$ esse estresse oxidativo é sistêmico e pode causar danos a toda a via neuromuscular.

Diferentemente desses resultados, três estudos ${ }^{17-19}$ não observaram fraqueza muscular respiratória de pacientes com AOS. Shepherd et al. ${ }^{19}$ hipotetizaram que a preservação da força dos músculos inspiratórios seria uma consequência do "treinamento" a que esses músculos são expostos, uma vez que estão em constante atividade durante os esforços inspiratórios. Já Tassinari et al. ${ }^{17}$ alegam que os resultados encontrados são esperados, já que a fisiopatologia da AOS está relacionada à VAS.

Não se tem certeza dos reais motivos desses resultados inconclusivos. Ainda assim, podemos presumir que a razão está na diversidade metodológica, por exemplo, amostras com um grupo apresentando diferentes gravidades da AOS. Tal fato foi observado no estudo feito por Steier et al. ${ }^{18}$ em que em um grupo de 21 indivíduos, quatro deles apresentavam o quadro leve da doença, o que pode ter colaborado com a não observância da fraqueza muscular. Além disso, dois estudos que relataram fraqueza muscular inspiratória tinham amostras com indivíduos que apresentam comorbidades, sendo elas, a obesidade ${ }^{9}$ e a diabetes mellitus tipo $2^{10}$.

Evidências mostram que doenças como obesidade e diabetes são condições com potencial lesivo para a força muscular respiratória 20,21 , fato esse que pode ter influenciado os resultados dos estudos de Carvalho et al. ${ }^{9}$ e Rehling et al. ${ }^{10}$, respectivamente. Ressalta-se que o estudo de Carvalho et al. ${ }^{9}$ não apresentou grupo controle e os grupos controle e AOS do estudo de Rehling et al. ${ }^{10}$ apresentaram diferença significativa com relação à idade, sendo os indivíduos diabéticos com AOS mais velhos quando comparados aos indivíduos diabéticos sem AOS. Sabe-se que a idade avançada é um outro fator que pode impactar negativamente a força muscular respiratória. Segundo a revisão feita por Pessoa et al. ${ }^{22}$ a PI máx. tende a reduzir na faixa etária de 40 a 60 anos de idade e continua reduzindo progressivamente. Sendo assim, a idade pode ter sido um fator que colaborou com os resultados encontrados no estudo de Rehling et al. ${ }^{10}$.

Os estudos em sua maioria utilizaram em suas amostras indivíduos com AOS em estágios mais graves da doença, sendo que somente um estudo incluiu indivíduos com AOS leve ${ }^{18}$, mesmo assim, em quantidade pequena, e um estudo ${ }^{14}$ não forneceu este dado. Tais resultados colaboram com a hipótese de que o comprometimento da força muscular respiratória pode estar associado à gravidade da doença.

Assim sendo, essa revisão apresenta que, estudos que relatam fraqueza muscular respiratória em pacientes com AOS demonstram que os músculos inspiratórios podem ser mais acometidos e que esse impacto pode estar relacionado aos estágios mais graves da doença, além da idade avançada e presença de outras comorbidades.
Diante dos resultados conflitantes encontrados na literatura, vale ressaltar a importância de se investigar a força muscular respiratória em grupos de pacientes com base na estratificação da AOS, incluindo casos leve, moderado e grave da doença, em busca de comprovação se a gravidade da doença influencia no comprometimento da função muscular, além de pesquisas que controlem os fatores de confusão.

O presente estudo apresenta algumas limitações como o número reduzido de estudos sobre o tema, bem como, estudos que trazem resultados conflitantes, provavelmente devido a diferentes características das amostras (idade, gravidade da AOS e comorbidades), que podem ser fatores confundidores.

\section{CONCLUSÃO}

Os resultados publicados na literatura ainda são divergentes com relação ao impacto da AOS na força muscular respiratória, no entanto, estudos que demonstram fraqueza muscular sugerem que a AOS compromete a força muscular inspiratória de pacientes com AOS moderada à grave. Dada a sua alta prevalência e importância clínica, faz-se necessário a realização de novas pesquisas que busquem entender melhor quais são os fatores determinantes do comprometimento da força muscular dessa população. Para isso, torna-se importante que as pesquisas estratifiquem os grupos estudados pelos fatores confundidores, tais como a faixa etária, a gravidade da AOS e as comorbidades dos participantes.

\section{FONTE DE FINANCIAMENTO}

Nada a declarar.

\section{CONFLITO DE INTERESSE}

Nada a declarar.

\section{REFERÊNCIAS}

1. American Academy of Sleep Medicine. Sleep-related breathing disorders in adults: recommendations for syndrome definition and measurement techniques in clinical research. The Report of an American Academy of Sleep Medicine Task Force. Sleep. 1999;22(5):667-89. http://dx.doi. org/10.1093/sleep/22.5.667. PMid:10450601.

2. Benjafield AV, Ayas NT, Eastwood PR, Heinzer R, Ip MSM, Morrell MJ, et al. Estimation of the global prevalence and burden of obstructive sleep apnoea: a literature-based analysis. Lancet Respir Med. 2019;7(8):687-98. http://dx.doi. org/10.1016/S2213-2600(19)30198-5. PMid:31300334.

3. Martins AB, Tufik S, Moura SMGPT. Síndrome da apnéiahipopnéia obstrutiva do sono. Fisiopatologia. J Bras Pneumol. 2007;33(1):93-100. http://dx.doi.org/10.1590/ S1806-37132007000100017. PMid:17568874.

4. Sunnetcioglu A, Sertogullarından B, Ozbay B, Gunbatar $H$, Ekin S. Obstructive sleep apnea related to rapid-eye- 
movement or non-rapid-eye-movement sleep: comparison of demographic, anthropometric, and polysomnographic features. J Bras Pneumol. 2016;42(1):48-54. http://dx.doi. org/10.1590/S1806-37562016000000012. PMid:26982041.

5. Palombini LO. Fisiopatologia dos distúrbios respiratórios do sono. J Bras Pneumol. 2010;36(Suppl. 2):4-9. http://dx.doi. org/10.1590/S1806-37132010001400003.

6. Junior CMC, Dal-Fabbro C, Bruin VMS, Tufik S, Bittencourt LRA. Consenso brasileiro de ronco e apneia do sono aspectos de interesse aos ortodontistas. Dental Press J Orthod. 2011;16(1):34.e1-10. http://dx.doi.org/10.1590/ S2176-94512011000100007.

7. Patil SP, Ayappa IA, Caples SM, Kimoff RJ, Patel SR, Harrod CG. Treatment of adult obstructive sleep apnea with positive airway pressure: An american academy of sleep medicine clinical practice guideline. J Clin Sleep Med. 2019;15(2):33543. http://dx.doi.org/10.5664/jcsm.7640. PMid:30736887.

8. McEvoy RD, Antic NA, Heeley E, Luo Y, Ou Q, Zhang X, et al. CPAP for prevention of cardiovascular events in obstructive sleep apnea. N EngIJ Med. 2016 Set 8;375(10):919-31. http:// dx.doi.org/10.1056/NEJMoa1606599. PMid:27571048.

9. Carvalho TMDCS, Soares AF, Climaco DCS, Secundo IV, Lima AMJ. Associação entre função pulmonar, força muscular respiratória e capacidade funcional de exercício em indivíduos obesos com síndrome da apneia obstrutiva do sono. J Bras Pneumol. 2018;44(4):279-84. http://dx.doi. org/10.1590/s1806-37562017000000031. PMid:29947714.

10. Rehling T, Banghoj AM, Kristiansen MH, Tarnow L, Molsted S. Reduced inspiratory muscle strength in patients with Type 2 Diabetes Mellitus and Obstructive Sleep Apnoea. J Diabetes Res. 2017;2017:4121794. http://dx.doi. org/10.1155/2017/4121794. PMid:29147664.

11. Chien MY, Wu YT, Lee PL, Chang YJ, Yang PC. Inspiratory muscle dysfunction in patients with severe obstructive sleep apnoea. Eur Respir J. 2010;35(2):373-80. http://dx.doi. org/10.1183/09031936.00190208. PMid:19643936.

12. WilcoxPG, Paré PD, Road JD, Fleetham JA. Respiratory muscle function during obstructive sleep apnea. Am Rev Respir Dis. 1990 apud Chien MY, Wu YT, Lee PL, Chang YJ, Yang PC. Inspiratory muscle dysfunction in patients with severe obstructive sleep apnoea. Eur Respir J. 2010;35(2):37380. http://dx.doi.org/10.1183/09031936.00190208. PMid:19643936.
13. Ferreira EVM. Musculatura respiratória: mitos e segredos. J Bras Pneumol. 2015;41(2):107-9. http://dx.doi.org/10.1590/ S1806-37132015000200002. PMid:25972964.

14. Caruso P, Albuquerque ALP, Santana PV, Cardenas LZ, Ferreira JG, Prina E, et al. Métodos diagnósticos para avaliação da força muscular inspiratória e expiratória. J Bras Pneumol. 2015;41(2):110-23. http://dx.doi.org/10.1590/ S1806-37132015000004474. PMid:25972965.

15. Barreiro E, Nowinski A, Gea J, Sliwinski P. Oxidative stress in the external intercostal muscles of patients with obstructive sleep apnoea. Thorax. 2007;62(12):1095-101. http://dx.doi. org/10.1136/thx.2006.069963. PMid:17573448.

16. Rosenzweig I, Glasser M, Polsek D, Leschziner GD, Williams SC, Morrell MJ. Sleep apnoea and the brain: a complex relationship. Lancet Respir Med. 2015;3(5):40414. http://dx.doi.org/10.1016/S2213-2600(15)00090-9. PMid:25887982.

17. Tassinari CCR, Piccin CF, Beck MC, Scapini F, Oliveira LCA, Signor LU, et al. Capacidade funcional e qualidade de vida entre sujeitos saudáveis e pacientes com apneia obstrutiva do sono. Medicina (B Aires). 2016;49(2):152-9. http://dx.doi. org/10.11606/issn.2176-7262.v49i2p152-159.

18. Steier J, Jolley CJ, Seymour J, Ward K, Luo YM, Polkey MI, et al. Increased load on the respiratory muscles in obstructive sleep apnea. Respir Physiol Neurobiol. 2010;171(1):54-60. http:// dx.doi.org/10.1016/j.resp.2010.01.012. PMid:20117253.

19. Shepherd KL, Jensen CM, Maddison KJ, Hillman DR, Eastwood PR. Relationship between upper airway and inspiratory pump muscle force in obstructive sleep apnea. Chest. 2006;130(6):1757-64. http://dx.doi.org/10.1378/ chest.130.6.1757. PMid:17166993.

20. Salome CM, King GG, Berend N. Physiology of obesity and effects on lung function. J Appl Physiol. 2010;108(1):20611. http://dx.doi.org/10.1152/japplphysiol.00694.2009. PMid:19875713.

21. Corrêa AP, Ribeiro JP, Balzan FM, Mundstock L, Ferlin EL, Moraes RS. Inspiratory muscle training in type 2 diabetes with inspiratory muscle weakness. Med Sci Sports Exerc. 2011;43(7):1135-41. http://dx.doi.org/10.1249/ MSS.0b013e31820a7c12. PMid:21200342.

22. Pessoa IMBS, Parreira VF, Fregonezi GA, Sheel AW, Chung F, Reid WD. Reference values for maximal inspiratory pressure: a systematic review. Can Respir J. 2014;21(1):43-50. http:// dx.doi.org/10.1155/2014/982374. PMid:24137574. 